\title{
MOBILE LEARNING EN EL GRADO DE EDUCACIÓN INFANTIL. UNA BUENA PRÁCTICA EN LA UNIVERSIDAD DE CANTABRIA
}

Mobile learning in the grade of children education. A good practice in the University of Cantabria

Aprendizagem móvel no grau da educação infantil. Boas práticas a Universidade de Cantabria

\section{Natalia González-Fernández (1) \\ Irina Salcines-Talledo (2)}

(1) Universidad de Cantabria, España. Teléfono: +34942201270. Correo electrónico: gonzalen@unican.es.

(2) Universidad de Cantabria, España. Teléfono: +34942201883. Correo electrónico: salcinesi@unican.es

\begin{abstract}
Resumen
Esta comunicación presenta una buena práctica de Mobile Learning, mediante el uso del Smartphone, desarrollada en la asignatura de Investigación e Innovación Educativa, en el Grado en Educación Infantil de la Universidad de Cantabria. Se trata de una experiencia en la que se promueve el uso académico de aplicaciones móviles de forma cooperativa para favorecer la evaluación formativa y compartida. En esta experiencia han participado 98 estudiantes de $4^{\circ}$ de Grado cuyas valoraciones han sido muy positivas, al incrementar su motivación, participación y autorregulación del aprendizaje. Generando un clima de aula en el que se contagiaban las ganas por seguir profundizando en el dominio de la materia.

Palabras clave: Evaluación formativa; Mobile Learning; Grado Educación Infantil; Smartphone
\end{abstract}

\begin{abstract}
This communication presents a good practice of Mobile Learning, through the use of the Smartphone, developed in the subject of Research in Innovation in Education of the Degree in Infant Education of the University of Cantabria. It is an experience in which
\end{abstract}


the academic use of mobile applications is promoted in a cooperative way to favor the formative and shared evaluation. In this experience, 98 students from 4th grade participated whose assessments have been very positive, increasing their motivation, participation and self-regulation of learning. Generating a classroom climate in which they felt the desire to continue to deepen the mastery of the subject.

Keywords: Training Assessment; Mobile Learning; Early Childhood Education; Smartphone

\section{Resumo}

Esta comunicação apresenta uma boa prática de Mobile Learning, usando o Smartphone, desenvolvida no curso de Investigação e Inovação Educativa na Educação Infantil Grau dela Universidade de Cantabria. É uma experiência em que o uso acadêmico de aplicações móveis é promovido em cooperação para promover a formação e avaliação compartilhada. Neste experimento 98 alunos participaram 4 a Série cujas avaliações foram muito positivas, aumentando a sua motivação, participação e aprendizagem auto-regulada. Criando um ambiente de sala de aula em que o desejo foram infectados por aprofundar ainda mais o domínio do assunto.

Palavras-chave: Avaliação formativa; Aprendizagem Mobile; Early Childhood Education Grau; Smartphone

\section{Introducción}

El contexto de Educación Superior ha cambiado considerablemente y la presencia de las tecnologías en él es incuestionable. Internet, y especialmente la denominada Web 2.0, ha trastocado las reglas de juego tradicionales de elaboración, distribución y consumo de la cultura (Area \& Pessoa, 2012). Ordenadores portátiles, tablets, teléfonos móviles, iPods y otros instrumentos han comenzado a formar parte de las dinámicas de clase (Marciales, 2012). Los entornos BYOD (Bring Your Own Device) han permitido solventar alguno de los problemas que tenía el profesorado a la hora de realizar procesos de evaluación formativa. Uno de ellos era la dificultad para recoger evidencias de las evaluaciones, generando una gran cantidad de papeles. Las aplicaciones nos permiten registrar automáticamente evaluaciones frecuentes, tanto del aprendizaje como del 
proceso, y en diferentes direcciones, autoevaluación, heteroevaluación y coevaluación. Concretamente aplicaciones como: Kahoot, Mentimeter y Edpuzzle.

El informe realizado por la Fundación Telefónica (2016) señala que el 82.3\% de los internautas utilizan el Smartphone como dispositivo para sus conexiones, seguido del ordenador (81.2\%) y la Tablet (34.4\%). En la misma línea, Ericsson (2016) prevé que para el año 2021 habrá en el mundo 9 mil millones de suscripciones móviles, 7.700 millones de banda ancha móvil y 6,3 mil millones de Smartphones.

Según Brazuelo y Gallego (2011), podemos definir el Mobile Learning como la modalidad educativa que facilita la construcción del conocimiento, la resolución de problemas de aprendizaje y el desarrollo de destrezas o habilidades diversas de forma autónoma y ubicua gracias a la mediación de dispositivos móviles portables. Presenta las siguientes características: flexibilidad, dado que se adapta a las necesidades de cada estudiante; portabilidad, puesto que su tamaño permite la movilidad con el usuario; inmediatez, posibilitando el acceso a la información en cualquier momento y, motivación, puesto que estimula al alumnado potenciando en él un papel más activo antela tarea de búsqueda, selección y análisis de la información en red.

Diversas investigaciones han demostrado los beneficios pedagógicos de introducir estos dispositivos móviles en las aulas, debido a las citadas características (Brazuelo \& Gallego, 2011; Corbeil \& Valdes-Corbeil, 2007; Hernández, 2009; ISEA, 2009; JISC infoNet, 2012; Marcos, Tamez \& Lozano, 2009; Palazón, 2015; Ramos, Herrera \& Ramírez, 2010; Sevillano, 2013; UNESCO, 2013; Villalonga \& MartaLozano, 2015). Ante esta realidad, nos planteamos introducir el Mobile Learning en determinados momentos de nuestra asignatura. A la propuesta de buena práctica del curso 2015-2016,basada en la introducción del Modelo Flipped Classroom, este curso hemos diseñado y añadido actividades de aprendizaje y evaluación formativa basadas en el Mobile Learning, concretamente adaptadas y apoyadas en una serie de Apps que explicaremos a continuación.

\section{Contextualización}

Esta experiencia, en la que han participado de forma constante y activa 98 estudiantes, se ha llevado a cabo en la Universidad de Cantabria durante el curso 2016-17 en el Grado de Educación Infantil. Concretamente, se ha aplicado en la asignatura de Investigación e Innovación Educativa de $4^{\circ}$ curso. 


\section{Diseño y desarrollo}

A lo largo de los ocho temas en los que se ha estructurado el contenido teórico-práctico de la asignatura, se han diseñado actividades de dificultad creciente, para las que nos hemos basado en la filosofía del Mobile Learning. Concretamente, hemos llevado a la práctica dicha modalidad de enseñanza, aprendizaje y evaluación formativa a través de tres fases que se han ido sucediendo cíclicamente para cada uno de los temas:

- $1^{\text {a }}$ FASE. Evaluación de conocimientos previos (Mentimeter). Al inicio de cada uno de los temas de la asignatura, hemos empleado la aplicación móvil “Mentimeter” lo que nos ha permitido recoger de forma inmediata, visual y anónima, los conocimientos previos con los que acceden a cada tema el grupo de estudiantes.

- $2^{a}$ FASE. Evaluación de la comprensión y de la relación con los conocimientos recientemente trabajados en la asignatura (EdPuzzle). En el desarrollo de cada uno de los temas, se propone la visualización reflexiva, analítica y crítica de un documento audiovisual, previamente creado, fracturado y cuestionado por las profesoras, de tal manera que el alumnado pueda enfrentarse a un reto cognitivo estimulante, afrontar su contenido y resolver las cuestiones planteadas.

- $3^{a}$ FASE. Evaluación de la comprensión de los contenidos teórico-prácticos desarrollados en cada uno de los temas (Kahoot). El día siguiente a la finalización de cada uno de los temas teórico-prácticos, se propone un concurso, mediante la aplicación Kahoot, en el que los estudiantes de forma grupal deben responder a diferentes cuestiones relativas al tema abordado en un tiempo determinado, relacionando, seleccionando y consensuando en el equipo las respuestas correctas.

Las tres aplicaciones seleccionadas, permiten ofrecer un feedback inmediato al alumnado, para que ellos sean conscientes de sus conocimientos y necesidades formativas y en consecuencia, puedan autorregular su proceso de aprendizaje conscientemente.

\section{Evaluación}

La evaluación de la buena práctica de Mobile Learning, se realiza analizando las respuestas obtenidas en el cuestionario de autopercepción del Mobile Learning, cumplimentado por los estudiantes al finalizar la asignatura. 
Hay que tener en cuenta que las actividades específicas propuestas mediante estas aplicaciones del Smartphone no han ido asociadas a una calificación numérica, con peso en la evaluación final.

Siguiendo la filosofía de la evaluación formativa, se han empleado como un sistema idóneo que favorece la mejora de los procesos de enseñanza, aprendizaje y evaluación, orientando y retroalimentando a los estudiantes para que, ellos mismos, reflexionen y emprendan acciones precisas que les permitan optimizar sus aprendizajes (Romero-Martín, Castejón-Oliva \& López-Pastor, 2015).

\section{Conclusiones}

Podemos apuntar como primeras conclusiones que los estudiantes valoran positivamente el uso académico de las citadas aplicaciones del Smartphone, debido al aumento de su motivación, participación y autonomía, al recibir una retroalimentación inmediata una vez cerrada la App, tanto del grupo de compañeros como del profesor. Esta retroalimentación se lleva a cabo de forma oral en el aula y, a través de cada uno de los blog diseñados por los grupos de trabajo cooperativo de los estudiantes.

Sin embargo, también nos hemos encontrado con estudiantes que,una vez finalizada la actividad pautada por los docentes, permanecen utilizando su dispositivo móvil con finalidades no académicas lo que provoca una dispersión en su atención.

\section{Referencias}

Area, M., \& Pessoa, T. (2012). De lo sólido a lo líquido: las nuevas alfabetizaciones ante los cambios culturales de la Web 2.0. Comunicar, 38, 13-20.

Brazuelo, F., \& Gallego, D. J. (2011). Mobile Learning. Los dispositivos móviles como recurso educativo. Sevilla: Editorial MAD.

Corbeil, J.R., \& Valdes-Corbeil, M.E. (2007). Are you ready for Mobile Learning? EducauseQuarterly, 2, 51- 58.

Fundación Telefónica (2016). La Sociedad de la Información en España 2015. Madrid: Fundación Telefónica.

Hernández, T. (2009). Educación sin tiempo: ¿M-learning o U-learning en la Investigación $\quad y \quad$ Docencia? Recuperado de: http://encuentrointernacional.ead.urbe.edu/2009/pdf/ponencias/03.pdf 
ISEA (2009). Mobile Learning, Análisis prospectivo de las potencialidades asociadas al Mobile Learning. ISEA. Recuperado de: http://www.iseamcc.net/eISEA/Vigilancia_tecnologica/informe_4.pdf

JISC infoNet (2012). InfoKit de aprendizaje móvil. Recuperado de: http://goo.gl/n0JLRZ

Marciales, G.P. (2012). Competencia informacional y brecha digital: preguntas y problemas emergentes derivados de la investigación. Nómadas, 36, 127-142.

Marcos, L., Tamez, R., \& Lozano, A. (2009). Aprendizaje móvil y desarrollo de habilidades en foros asincrónicos de comunicación. Comunicar, 33, 93-100.

Palazón, J. (2015). Aprendizaje móvil basado en microcontenidos como apoyo a la interpretación instrumental en el aula de música en secundaria. Pixel-Bit. Revista de Medios de Comunicación, 46, 119-136.

Ramos, A.I., Herrera, J.A., \& Ramírez, M.S. (2010). Desarrollo de habilidades cognitivas con aprendizaje móvil: un estudio de casos. Comunicar,34, 201-209. doi: 10.3916/C34-2010-03-20

Romero-Martín, R.; Castejón-Oliva, F.J., \& López-Pastor, V. (2015). Divergencias del alumnado y del profesorado universitario sobre las dificultades para aplicar la evaluación formativa. RELIEVE, 21(1), 1-66. DOI:10.7203/relieve.21.1.5169

Sevillano, M.L. (2013). Enseñanza y aprendizaje con dispositivos móviles. En J. I. Aguaded y J. Cabero (coord.), Tecnologías y Medios para la Educación en la eSociedad (pp.159-184). Madrid: Alianza Editorial.

UNESCO (2013). El futuro del aprendizaje móvil. Implicaciones para la planificación y la formulación de políticas. París: Organización de las Naciones Unidas para la Educación, la Ciencia y la Cultura. Recuperado de: http://goo.gl/ywtmyo

Villalonga, C., \& Marta-Lozano, C. (2015). Modelo de integración educomunicativa de “apps” móviles para la enseñanza y aprendizaje. Píxel-Bit, 46, 137-153. 